\section{Should All Patients 75 Years of Age or Older Receive Intensive Management for Hypertension?}

\section{THE "PRO" SIDE}

It is much more important to know what sort of a patient has a disease than what sort of a disease a patient has. - Sir William Osler (1849-1919)

The Canadian population is aging, with an estimated onequarter of Canadians reaching 65 years of age or older by 2036, and $25 \%$ of those individuals being 80 years and older. ${ }^{1}$ Among the leading causes of death in Canada for those aged 75-84 are cardiovascular and cerebrovascular diseases. ${ }^{2}$ Globally, high blood pressure is the leading risk factor for death and disability. ${ }^{3}$

To evaluate whether lower targets for systolic blood pressure (BP) could further protect against cardiovascular disease, SPRINT (the Systolic Blood Pressure Intervention Trial) compared a standard systolic BP target of $140 \mathrm{~mm} \mathrm{Hg}$ with an intensive target of $120 \mathrm{~mm} \mathrm{Hg}$. The overall trial, which enrolled almost 10000 participants, showed that treating to an intensive systolic BP target reduced fatal and nonfatal cardiovascular events from $6.8 \%$ to $5.2 \%$ (hazard ratio 0.75 , absolute risk reduction [ARR] 1.6\%, number needed to treat [NNT] 63) over 3.3 years. All-cause mortality was also reduced (ARR 1.2\%, NNT 84 over 3.3 years). ${ }^{4}$

A closer look at the design of SPRINT reveals that it was intended to examine high-risk populations and to specifically recruit individuals within those groups, including individuals aged 75 or older. Patients were eligible to participate if they met one of the following inclusion criteria: history of clinical or subclinical cardiovascular disease, chronic kidney disease, 10-year Framingham cardiovascular disease risk of $15 \%$ or more, or age 75 or older. Patients were excluded if they had systolic BP less than $110 \mathrm{~mm} \mathrm{Hg}$ following a minute of standing, expected survival of less than 3 years, diabetes mellitus, or heart failure, or if they lived in a nursing home. Frailty and lower functional status were not specified as exclusion criteria. In total, $28 \%$ of participants recruited $(n=2636)$ met the inclusion criteria of age 75 and older, with an average age of about 80 years in this subgroup. Of these, more than $80 \%$ were characterized as "less fit" (about 55\%) or "frail" (about 31\%) according to a validated frailty index. The results, including outcomes based on frailty, were evaluated separately. Importantly, the results for this prespecified subgroup were more impressive than the results of the overall trial. The NNT over 3.3 years for the primary outcome (a composite of myocardial infarction, acute coronary syndrome, stroke, heart failure, or death from cardiovascular causes) was lower within this group than for the overall trial (27 versus 63, respectively), and the NNT for all-cause mortality was also lower (41 versus 84 , respectively). These benefits were consistent regardless of frailty status, with the frail patients and slowest walkers benefiting as much as younger, fitter participants. ${ }^{5}$ Additionally, this outcome was achieved with an average of 3 antihypertensive medications, compared with 2 in the standard treatment arm, which indicated that this population had hypertension that was responsive to treatment (i.e., nonresistant hypertension).

The intensive systolic BP achieved in those 75 years and older was slightly higher $(123.4 \mathrm{~mm} \mathrm{Hg})$ compared to that achieved with intensive treatment in all trial participants $(121.4 \mathrm{~mm} \mathrm{Hg})$; these values can be compared to the systolic BP target achieved with standard treatment $(134.8 \mathrm{~mm} \mathrm{Hg})$. Furthermore, within the intensive treatment group, mean systolic BP during follow-up was slightly higher for participants classified as less fit $(123.3 \mathrm{~mm} \mathrm{Hg})$ or frail (124.3 $\mathrm{mm} \mathrm{Hg}$ ) than for those considered to be fit $(121.4 \mathrm{~mm} \mathrm{Hg}$ ). Overall, the difference in systolic BP between treatment groups ranged from 10.8 to $13.5 \mathrm{~mm} \mathrm{Hg}$. These BP values were obtained with an unattended automated cuff, also called an automated office blood pressure device. ${ }^{4}$ For clinicians using a BP measurement device that is attended and/or manual, the systolic BP reading may be 5 to 10 $\mathrm{mm} \mathrm{Hg}$ higher. Achieving these BP targets is contingent on using a similar method for BP measurement. ${ }^{6}$

To explore a preventive role in cognitive impairment, a subgroup analysis was planned a priori to evaluate the effect of intensive lowering of systolic BP on probable dementia and mild cognitive impairment. ${ }^{7}$ The incidence of probable dementia was not decreased significantly (although the trend was toward reduction), but there was a significant 19\% relative risk reduction (RRR) in mild cognitive impairment, with an RRR of $15 \%$ for the composite end point of probable dementia or mild cognitive impairment. Although concerns have previously been expressed that lower BP causes hypoperfusion of the brain, leading to negative effects, this problem was not observed. It is promising that, over 3.3 years of intervention and just over 5 years of follow-up, mild cognitive impairment was reduced. The trial was stopped early (after 3.3 years) because of positive cardiovascular effects in the intensive arm, truncating the ability to assess these cognitive outcomes, which typically manifest slowly over many years. ${ }^{7}$ 
Importantly, the oldest subgroup tolerated the intensive treatment to the same degree as the standard treatment. Serious adverse events were defined as events that were fatal or life-threatening, resulting in significant or persistent disability, requiring or prolonging a hospitalization, or being significant enough to require intervention. The outcomes evaluated included hypotension, syncope, electrolyte abnormalities, and acute kidney injury or renal failure. The incidence of serious adverse events was virtually identical in the 2 treatment arms (48.4\% versus $48.3 \%$ in the standard treatment arm), with none of the individual outcomes having a statistically significant difference. There was also no difference in injurious falls between the groups, a finding that was independent of frailty status. Typically, risks of orthostatic hypotension and falls are cited as reasons for not intensifying hypertension management, and these findings provide reassurance that these outcomes did not differ with the BP target. While there was an overall increase in the rate of adverse events, they were mostly events that could be addressed and that should not have long-term consequences. For this reason, a monitoring plan is important, so that adjustments, like deprescribing, can be made on the basis of treatment response. ${ }^{5}$

On the basis of results from the Hypertension in the Very Elderly Trial, Hypertension Canada previously recommended that those over 80 years of age be treated to a target of less than $150 \mathrm{~mm} \mathrm{Hg}$. In response to SPRINT, Hypertension Canada has adopted BP targets based on risk level, and has abandoned recommendations based on age alone. ${ }^{8}$ Other countries have done the same. The US guidelines were changed in 2018 to recommend a lower systolic BP targetless than $130 \mathrm{~mm} \mathrm{Hg}$ - for all high-risk patients.?

Ultimately, these results show meaningful benefit in those aged 75 and above, and were achieved with relative ease, by adding one more antihypertensive medication to their regimen. Moreover, the results were obtained with a side effect profile that was no different from that associated with standard BP treatment targets. As with many treatment decisions for elderly patients, the benefits (specifically, decreases in cardiovascular disease and mortality that are not achieved with many therapies for this age group) need to be weighed against the risk tolerance for additional monitoring and medications. To maximize treatment benefit and minimize harms, the goal should be to work in partnership with patients (especially those 75 years of age or older) to treat their hypertension to evidence-based guideline recommendations, with a monitoring plan in place. It seems reasonable to give this strategy a try in patients who are willing and able.

\section{References}

1. Population by age and sex, 2013. Ottawa (ON): Statistics Canada; [cited 2019 Mar 4]. Available from: https://www150.statcan.gc.ca/n1/pub/91-215-x/ 2013002/part-partie2-eng.htm

2. Leading causes of death, total population, by age group, 2016. Ottawa (ON): Statistics Canada; [cited 2019 Mar 4]. Available from: https:// www150.statcan.gc.ca/t1/tbl1/en/tv.action?pid $=1310039401 \&$ pick Members\%5B0\%5D $=2.28$ \&pickMembers\%5B1\%5D=3.1

3. Lim SS, Vos T, Flaxman AD, Danaei G, Shibuya K, Adair-Rohani H, et al. A comparative risk assessment of burden of disease and injury attributable to 67 risk factors and risk factor clusters in 21 regions, 1990-2010: a systematic analysis for the Global Burden of Disease Study 2010. Lancet. 2012;380 (9859):2224-60.

4. SPRINT Research Group; Wright JT Jr, Williamson JD, Whelton PK, Snyder $\mathrm{JK}$, Sink KM, Rocco MV, et al. A randomized trial of intensive versus standard blood-pressure control. N Engl J Med. 2015;373(22):2103-16.

5. Williamson JD, Supiano MA, Applegate WB, Berlowitz DR, Campbell RC, Chertow GM, et al. Intensive vs standard blood pressure control and cardiovascular disease outcomes in adults aged $\geq 75$ years: a randomized clinical trial. JAMA. 2016;315(24):2673-82.

6. Bakris GL. The implications of blood pressure measurement methods on treatment targets for blood pressure. Circulation. 2016;134(13):904-5.

7. SPRINT MIND Investigators for SPRINT Research Group; Williamson JD, Pajewski NM, Auchus AP, Bryan RN, Chelune G, Cheung AK, et al. Effect of intensive vs standard blood pressure control on probable dementia: a randomized clinical trial. JAMA. 2019;321(6):553-61.

8. Nerenberg KA, Zarnke KB, Leung AA, Dasgupta K, Butalia S, McBrien K, et al. Hypertension Canada’s 2018 guidelines for diagnosis, risk assessment, prevention, and treatment of hypertension in adults and children. Can J Cardiol. 2018;34(5):506-25.

9. Whelton PK, Carey RM, Aronow WS, Casey DE Jr, Collins KJ, Dennison Himmelfarb C, et al. 2017 ACC/AHA/AAPA/ABC/ACPM/AGS/APhA/ASH/ ASPC/NMA/PCNA guideline for the prevention, detection, evaluation, and management of high blood pressure in adults: executive summary: a report of the American College of Cardiology/American Heart Association Task Force on Clinical Practice Guidelines. Hypertension. 2018;71(6):1269-324.

Ann Thompson, BScPharm, PharmD, ACPR

Faculty of Pharmacy and Pharmaceutical Sciences University of Alberta

Edmonton, Alberta

Competing interests: None declared.

See page 251 for the "Con" side of this debate. 


\section{THE "CON" SIDE}

Optimal blood pressure (BP) has been debated since hypertension was first identified more than 100 years ago as a cardiovascular risk factor. Previous trials have shown that reducing BP in elderly patients is beneficial. ${ }^{1-3}$ However, contrary to what golfers and limbo enthusiasts alike strive to achieve, lower - in the context of BP — is not always better. Two trials involving elderly Japanese patients (mean age 74 and 76 years, respectively) with a systolic BP above $160 \mathrm{~mm} \mathrm{Hg}$ both demonstrated that "strict" BP control (systolic BP $<140 \mathrm{~mm} \mathrm{Hg}$ ) was not superior to "moderate" control (systolic BP $140-159 \mathrm{~mm} \mathrm{Hg}$ ) with respect to adverse cardiovascular and renal events. ${ }^{4.5} \mathrm{~A}$ subsequent meta-analysis assessed a BP target of $<160$ / $<90 \mathrm{~mm} \mathrm{Hg}$ versus $<140 /<90 \mathrm{~mm} \mathrm{Hg}$ in adults 65 years of age or older who had hypertension and found no difference in all-cause death and cardiovascular serious adverse events. ${ }^{6}$ The latest contribution to this debate is the Systolic Blood Pressure Intervention Trial (SPRINT), the seminal contemporary hypertension trial that has brought about countless metaphorical presentation titles at conferences worldwide. In that spirit, when it comes to intensive hypertension management in older adults, I would argue that we should walk rather than "SPRINT" toward a benefit.

In SPRINT, an "intensive" systolic BP target $(<120 \mathrm{~mm} \mathrm{Hg})$ was compared with a "standard" target $(<140 \mathrm{~mm} \mathrm{Hg})$ in 9361 patients (mean age 68 years, 25\% women) with initial systolic BP between 130 and $180 \mathrm{~mm} \mathrm{Hg}{ }^{7}$ After 3.3 years, patients in the group with intensive systolic $\mathrm{BP}$ target had a lower rate of the primary composite end point of cardiovascular death, acute coronary syndrome, stroke, and heart failure (absolute reduction 1.6\%; number needed to treat $[\mathrm{NNT}]$ 63). Although intensive treatment also lowered the risk of all-cause death (NNT 84), heart failure (NNT 125), and cardiovascular death (NNT 167), it did not reduce myocardial infarction or stroke. Intensive treatment was not without risk-it increased certain serious adverse events, including acute kidney injury or renal failure (number needed to harm $[\mathrm{NNH}] 56$ ), hypotension (NNH 100), electrolyte abnormalities (NNH 125), and syncope (NNH 167). It is important to note that each of these was a serious adverse event, defined as "an event that was fatal or life-threatening, resulting in significant or persistent disability, requiring or prolonging a hospitalization, or was an important medical event that the investigator judged to be a significant hazard or harm to the participant."

A preplanned subgroup analysis of SPRINT ${ }^{8}$ included 2636 patients 75 years of age or older (mean age 80 years, $38 \%$ women), which constituted only $28 \%$ of the overall population. The results were similar to those of the overall trial: intensive treatment reduced the primary end point (NNT 29), all-cause death (NNT 39), and heart failure (NNT 67), yet did not lower the risk of cardiovascular death, myocardial infarction, or stroke. Interestingly, the primary end point was not reduced in the subgroup of patients younger than 75 years of age. 7 In the subgroup of patients 75 years of age or older, treatment-related serious adverse events were numerically (though not significantly) higher with intensive treatment. ${ }^{8}$ From an evidencebased purist perspective, a subgroup analysis—one that excluded $72 \%$ of the study population—should be viewed with skepticism. ${ }^{9}$ One could argue that such an analysis should be used only to generate future research, not to dictate clinical practice. With respect to other prespecified subgroups, the primary end point was lower in patients without (but not with) chronic kidney disease, in those without (but not with) cardiovascular disease, in men (but not women), and in patients with a baseline systolic BP of $132 \mathrm{~mm} \mathrm{Hg}$ or below (but not $133-144 \mathrm{~mm} \mathrm{Hg}$ or $\geq 145 \mathrm{~mm} \mathrm{Hg}$ ). As any angler will agree, if you go fishing enough times, yoưre bound to catch something.

I concede that the results of SPRINT are impressive. Notwithstanding, the argument against intensive BP management in older persons is less about critical appraisal and more about the pragmatic application of these data in practice. It is not so much about, "What does the evidence show?" but rather, "How does this evidence apply to my patient?"

The SPRINT was conducted in a relatively healthy population. It excluded patients with diabetes mellitus or previous stroke, as well as many frail older patients, specifically those with a 1-min standing systolic BP below $110 \mathrm{~mm} \mathrm{Hg}$, proteinuria (24-h urinary protein excretion $\geq 1 \mathrm{~g} /$ day or similar), estimated glomerular filtration rate (eGFR) below $20 \mathrm{~mL} \mathrm{~min}{ }^{-1} 1.73 \mathrm{~m}^{-2}$ or end-stage renal disease, left ventricular ejection fraction below $35 \%$, life expectancy less than 3 years, dementia, or residence in a nursing home. Even among those aged 75 years or older, only $25 \%$ had a history of cardiovascular disease and only $16 \%$ had eGFR below $45 \mathrm{~mL} \mathrm{~min}^{-1} 1.73 \mathrm{~m}^{-2}$. At baseline, mean BP was $142 / 71 \mathrm{~mm} \mathrm{Hg}$, and patients were taking an average of 2 antihypertensive agents. Also, it was challenging to achieve systolic BP below $120 \mathrm{~mm} \mathrm{Hg}$ even with a trial protocol: mean systolic BP in the intensive treatment group over the followup period was $123 \mathrm{~mm} \mathrm{Hg}$. Furthermore, given that the trial was discontinued prematurely, the long-term effect of intensive BP management remains unknown. There was no difference in the rate of injurious falls (i.e., falls that resulted in hospital admission or evaluation in an emergency department), but the total number of falls was not reported. However, in older persons, a non-injurious fall or even the fear of falling may have a negative impact on quality of life. In addition, although the rate of syncope was similar between groups, data were not reported for symptomatic lightheadedness, which also can have a subtle deleterious effect on patients' daily activities.

Some clinicians may argue that intensive BP treatment has an overall net clinical benefit. However, this conclusion disregards the values that patients assign to those outcomes. Many older persons value quality over quantity of life. Thus, some patients may place more value on avoiding the possible adverse effects of antihypertensive therapy than on the relatively small reduction in all-cause mortality. Furthermore, health literacy may be low among elderly patients. Therefore, it is imperative to employ novel ways of engaging patients and their families in shared decision-making to determine whether intensive BP management aligns with their health goals. Other 
practical considerations include the risk of nonadherence, potential contribution to polypharmacy, and cost.

I believe that the SPRINT results should have a meaningful impact on clinical practice, but would encourage clinicians not to let any bias favouring the efficacy data to negate consideration of patients' values and preferences. In my own admittedly anecdotal experience, when I have engaged in shared decision-making with select patients in my practice who meet the SPRINT criteria, most have declined to pursue a systolic BP target of less than $120 \mathrm{~mm} \mathrm{Hg}$. When it comes to $\mathrm{BP}$ control in older patients, the best advice is likely the often quoted, yet seldom followed medical axiom: treat the patient, not the number.

\section{References}

1. SHEP Cooperative Research Group. Prevention of stroke by antihypertensive drug treatment in older persons with isolated systolic hypertension. Final results of the Systolic Hypertension in the Elderly Program (SHEP). JAMA. 1991;265(24):3255-64.

2. Hansson L, Lindholm LH, Ekbom T, Dahlöf B, Lanke J, Scherstén B, et al. Randomised trial of old and new antihypertensive drugs in elderly patients: cardiovascular mortality and morbidity with Swedish Trial in Older Patients with Hypertension-2 study. Lancet. 1999;354(9192):1751-6.

3. Beckett NS, Peters R, Fletcher AE, Staessen JA, Liu L, Dumitrascu D, et al. Treatment of hypertension in patients 80 years of age or older. $N$ Engl J Med. 2008;358(18):1887-98.

4. JATOS Study Group. Principal results of the Japanese trial to assess optimal systolic blood pressure in elderly hypertensive patients (JATOS). Hypertens Res. 2008;31(12):2115-27.

5. Ogihara T, Saruta T, Rakugi H, Matsuoka H, Shimamoto K, Shimada K, et al. Target blood pressure for treatment of isolated systolic hypertension in the elderly: Valsartan in Elderly Isolated Systolic Hypertension Study. Hypertension. 2010;56(2):196-202.
6. Garrison SR, Kolber MR, Korownyk CS, McCracken RK, Heran BS, Allan GM. Blood pressure targets for hypertension in older adults. Cochrane Database Syst Rev. 2017;8:CD011575.

7. SPRINT Research Group; Wright JT Jr, Williamson JD, Whelton PK, Snyder JK, Sink KM, Rocco MV, et al. A randomized trial of intensive versus standard blood-pressure control. N Engl J Med. 2015;373(22):2103-16.

8. Williamson JD, Supiano MA, Applegate WB, Berlowitz DR, Campbell RC, Chertow GM, et al. Intensive vs standard blood pressure control and cardiovascular disease outcomes in adults aged $\geq 75$ years: a randomized clinical trial. JAMA. 2016;315(24):2673-82.

9. Sun X, Ioannidis JP, Agoritsas T, Alba AC, Guyatt G. How to use a subgroup analysis: users' guide to the medical literature. JAMA. 2014;311(4):405-11.

Arden R Barry, BSC, BSc(Pharm), PharmD, ACPR

Chilliwack General Hospital

Lower Mainland Pharmacy Services

Chilliwack, British Columbia

Faculty of Pharmaceutical Sciences

The University of British Columbia

Vancouver, British Columbia

Competing interests: None declared. 